\title{
EDITORIAL
}

\section{Can alcohol consumption be an alternative treatment for fibromyalgia?}

\author{
Mei Chung ${ }^{1}$ and Chenchen Wang ${ }^{2^{*}}$ \\ See related research by Kim et al. http://arthritis-research.com/content/15/2/R42
}

\begin{abstract}
Treatment of chronic pain conditions such as fibromyalgia is challenging due to limitations of drug therapies. An initial exploration into the relationships between self-reported alcohol consumption, symptom severity, and quality of life for individuals with fibromyalgia sheds new light on plausible hypotheses and potential mechanisms of action for future research. Evidence suggests that alcohol consumption may improve social and psychological factors because of activity in the ascending and descending pain pathways in modulating gamma-aminobutyric acid neurotransmission. Further methodologically rigorous studies in this field to improve well-being of individuals with fibromyalgia are warranted.
\end{abstract}

In a previous issue of Arthritis Research \& Therapy, Kim and colleagues [1] reported the first study to examine the association between alcohol consumption, symptom severity, and quality of life of individuals with fibromyalgia (FM). Treatment of chronic pain conditions such as FM is challenging because the drug therapies currently available are expensive and associated with numerous limitations such as undesirable side effects and addiction or tolerance issues. Despite undergoing drug treatment, patients are often left with unrelieved pain, restricted mobility, and reduced physical function. Lifestyle interventions, including dietary modifications such as alcohol consumption, have gained popularity as explorations in symptom management.

Several observational studies have examined the association between alcohol consumption and chronic pain conditions. In a prospective study, Bergman and colleagues [2] found that regular weekly or daily alcohol

\footnotetext{
*Correspondence: cwang2@tuftsmedicalcenter.org

${ }^{2}$ Division of Rheumatology, Tufts Medical Center, Tufts University School of Medicine, Box 406, Boston, MA 02111, USA

Full list of author information is available at the end of the article
}

consumption is a significant protective factor for the development of chronic pain. Two case-control studies have also shown that moderate alcohol consumption correlates with a reduced risk of rheumatoid arthritis $[3,4]$. However, a systematic review of nine epidemiological studies has concluded that alcohol consumption is not associated with low back pain [5]. Kim and colleagues are the first to examine the association between alcohol consumption and FM symptom severity and quality of life. Among the 946 adult FM patients (94\% women, mean age of 49 years old) reporting low or moderate alcohol consumption ( $\leq 3$ or $>3$ to 7 drinks per week), there was lower FM symptom severity and better quality-of-life scores compared with those who reported no alcohol consumption (non-drinkers). However, these associations were not observed in patients who were heavy drinkers ( $>7$ drinks per week) compared with non-drinkers. Drinkers had higher education, less unemployment, lower body mass index, and lower frequency of opioid use than non-drinkers. Thus, their analyses were adjusted for these potential confounders.

Because alcohol consumption consists of complex behaviors that intersect with many social, economic, psychological, and demographic factors, disentangling this complex web of relationships is a challenge. Interestingly, after exploring possible mechanisms for their findings, Kim and colleagues speculated that alcohol consumption may attenuate FM symptoms and improve quality of life by mediating psychological benefits and stress relief or by promoting factors associated with social integration. Another possible mechanism proposed is central nervous mediation via the modulating gamma-aminobutyric acid (GABA) system. Several neurotransmitters (including GABA) in the ascending and descending pain pathways have been implicated in FM [6]. Thus, behavioral and pharmacological therapies that modulate or mimic the effects of GABA production can be promising for FM treatment. 
This initial exploration into the relationships between alcohol consumption, symptom severity, and quality of life for FM has posed a number of questions that need to be answered by future studies with stronger study designs. Kim and colleagues discussed limitations associated with cross-sectional study design, and we would like to underscore additional concerns relating to the potential for non-random misclassifications of alcohol consumption among FM patients with different levels of symptom severity or quality of life. Such information bias is not uncommon in cross-sectional studies providing data on both exposure and outcome variables from questionnaires. Because the questionnaires are administered at the same time in a cross-sectional study and relied on respondent recall, the measurement errors of exposure (that is, alcohol consumption) may be dependent on the measurement errors of the outcomes (that is, quality of life or FM symptoms). If such dependent bias occurs, the observed association between exposure and outcome is likely to be inflated [7]. Owing to lack of prior data demonstrating that such bias exists in cross-sectional studies of alcohol consumption and FM symptom severity, we provide a possible scenario to examine the potential impacts of dependent bias on this relationship. FM patients who on one occasion had more severe symptoms may in fact have consumed more alcohol to ameliorate symptoms and thus may have felt better (fewer symptoms) at the time of the survey, or FM patients with more severe symptoms at the time of the survey may have stopped drinking alcohol (thus no symptom relief) because of their symptoms. The impact of such dependent bias would result in an inflation of the 'true' association. Owing to cross-sectional design, this potential bias cannot be evaluated. In addition to the potential for dependent bias, patients with FM may be too embarrassed to reveal their 'true' alcohol intake level because of social desirability biases [8]. Such reporting bias [9], however, is likely to be random and independent of symptom severity. There is still great uncertainty in the study results; therefore, we agree with Kim and colleagues that these preliminary results should not be used as grounds for advising patients to drink alcohol.

In sum, the study by Kim and colleagues sheds new light on plausible hypotheses and mechanisms to consider in future methodologically rigorous studies to improve the well-being of individuals with FM. Because it may not be feasible to randomize alcohol consumption in human subject research, methodologically rigorous prospective longitudinal studies are needed. Measuring alcohol consumption at different time points is essential in order to minimize dependent biases from patient-reported exposure and outcome measures.

\section{Abbreviations}

FM: Fibromyalgia; GABA: Gamma-aminobutyric acid

\section{Competing interests}

The authors declare that they have no competing interests.

\section{Acknowledgments}

$\mathrm{CW}$ is supported by awards from the National Center for Complementary and Alternative Medicine. The contents of this editorial are solely the responsibility of the authors and do not necessarily represent the official views of the National Center for Complementary and Alternative Medicine or the National Institutes of Health. The sponsors had no role in the design and conduct of the study; collection, management, analysis, and interpretation of the data; or preparation, review, or approval of the editorial.

\section{Author details}

${ }^{1}$ Nutrition/Infection Unit, Department of Public Health and Community Medicine, Tufts University School of Medicine, 150 Harrison Ave, Jaharis 264, Boston, MA 02111, USA. ²Division of Rheumatology, Tufts Medical Center, Tufts University School of Medicine, Box 406, Boston, MA 02111, USA.

\section{Published: 29 Nov 2013}

\section{References}

1. Kim CH, Vincent A, Clauw DJ, Luedtke CA, Thompson JM, Schneekloth TD, Oh TH: Association between alcohol consumption and symptom severity and quality of life in patients with fibromyalgia. Arthritis Res Ther 2013, 15:R42.

2. Bergman S, Herrström P, Jacobsson LT, Petersson IF: Chronic widespread pain: a three year follow-up of pain distribution and risk factors. J Rheumatol 2002, 29:818-825.

3. Maxwell JR, Gowers IR, Moore DJ, Wilson AG: Alcohol consumption is inversely associated with risk and severity of rheumatoid arthritis. Rheumatology (Oxford) 2010, 49:2140-2146.

4. Källberg H, Jacobsen S, Bengtsson C, Pedersen M, Padyukov L, Garred P, Frisch M, Karlson EW, Klareskog L, Alfredsson L: Alcohol consumption is associated with decreased risk of rheumatoid arthritis: results from two Scandinavian case-control studies. Ann Rheum Dis 2009, 68:222-227.

5. Leboeuf-Yde C: Alcohol and low-back pain: a systematic literature review. J Manipulative Physiol Ther 2000, 23:343-346.

6. Fitzgerald CT, Carter LP: Possible role for glutamic acid decarboxylase in fibromyalgia symptoms: a conceptual model for chronic pain. Med Hypotheses 2011, 77:409-415.

7. Kristensen P: Bias from nondifferential but dependent misclassification of exposure and outcome. Epidemiology 1992, 3:210-215.

8. Davis CG, Thake J, Vilhena N: Social desirability biases in self-reported alcohol consumption and harms. Addict Behav 2010, 35:302-311.

9. Midanik LT: Perspectives on the validity of self-reported alcohol use. Br J Addict 1989, 84:1419-1423.

\subsection{6/ar4394}

Cite this article as: Chung and Wang: Can alcohol consumption be an alternative treatment for fibromyalgia?. Arthritis Research \& Therapy 2013, 15:126 